\title{
Emission State Structure and Linewidth Broadening Mechanisms in Type-II CdSe/CdTe Core-Crown Nanoplatelets: A Combined Theoretical - Single Nanocrystal Optical Study
}

\section{Supporting Information}

\author{
Violette Steinmetz, ${ }^{\dagger}$ Juan I. Climente ${ }^{\ddagger}{ }^{\ddagger}$ Raj Pandya, ${ }^{\circledR}$ Josep Planelles, ${ }^{\ddagger}$ Florent \\ Margaillan, ${ }^{\dagger}$ Yuttapoom Puttisong, ${ }^{\S}$ Marion Dufour, " Sandrine Ithurria," Ashish \\ Sharma, ${ }^{\perp}$ Girish Lakhwani, ${ }^{\perp}$ Laurent Legrand, ${ }^{\dagger}$ Frédérick Bernardot,${ }^{\dagger}$ Christophe \\ Testelin $^{\dagger}{ }^{\dagger}$ Maria Chamarro, ${ }^{\dagger}$ Alex W. Chin ${ }^{\dagger}$ Akshay Rao, ${ }^{\top}$ and Thierry Barisien ${ }^{*} \dagger$ \\ †Sorbonne Université, Institut des NanoSciences de Paris, INSP, CNRS-UMR 7588, 4 \\ place Jussieu, F-75005, Paris, France
}

$\ddagger$ Departament de Química Física i Analítica, Universitat Jaume I, E-12080, Castelló de la Plana, Spain

IDepartment of Physics, Cavendish Laboratory, University of Cambridge, 19 JJ Thompson Avenue, Cambridge, CB3 OHE, UK

$\S$ Functional Electronic Materials, Department of Physics, Chemistry and Biology, Linköping University, 58183 Linköping, Sweden

|| Laboratoire de Physique et d'Étude des matériaux, ESPCI Paris, PSL Research University, CNRS UMR 8213, 10 rue Vauquelin, 75005 Paris, France $\perp A R C$ Centre of Excellence in Exciton Science, School of Chemistry, The University of Sydney, Sydney, New South Wales 2006, Australia

E-mail: barisien@insp.jussieu.fr 


\section{Table of contents (11 pages, 5 figures)}

\section{Structural and optical characterization $\ldots \ldots \ldots \ldots \ldots \ldots \ldots$ S2}

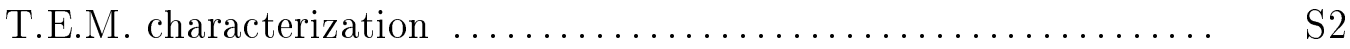

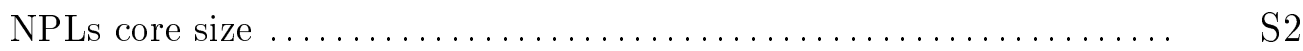

Large core and small core NPLs size $\ldots \ldots \ldots \ldots \ldots \ldots \ldots \ldots \ldots \ldots$. S3

Temperature dependence of the band gap $\ldots \ldots \ldots \ldots \ldots \ldots \ldots$ S4

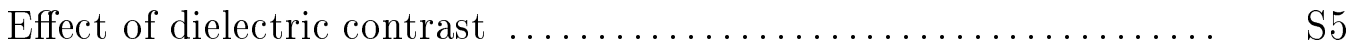

Low temperature Raman spectroscopy $\ldots \ldots \ldots \ldots \ldots \ldots \ldots \ldots \ldots$ S7

Huang-Rhys factor extraction $\ldots \ldots \ldots \ldots \ldots \ldots \ldots \ldots \ldots \ldots \ldots \ldots \ldots$

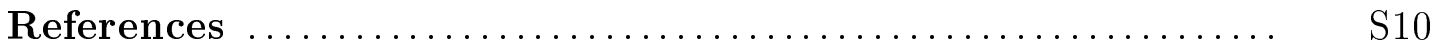




\section{Structural and optical characterization}

\section{T.E.M. characterization}

NPLs core size

(a)

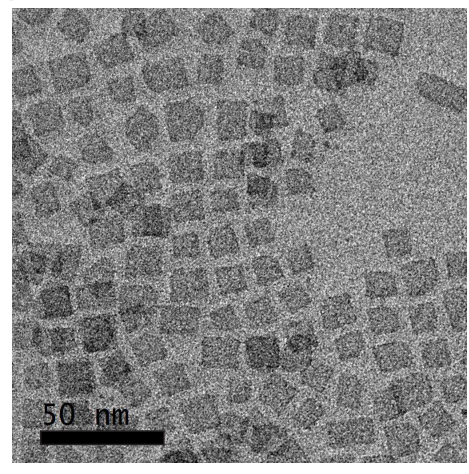

(c)

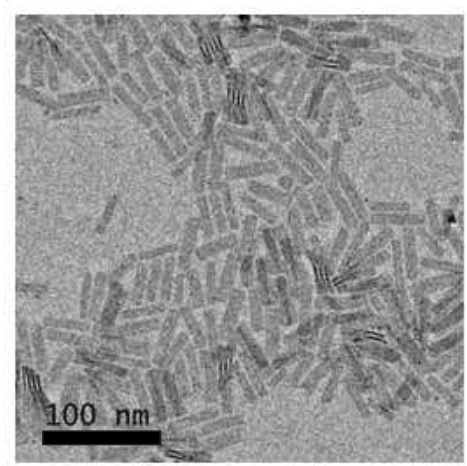

(b)

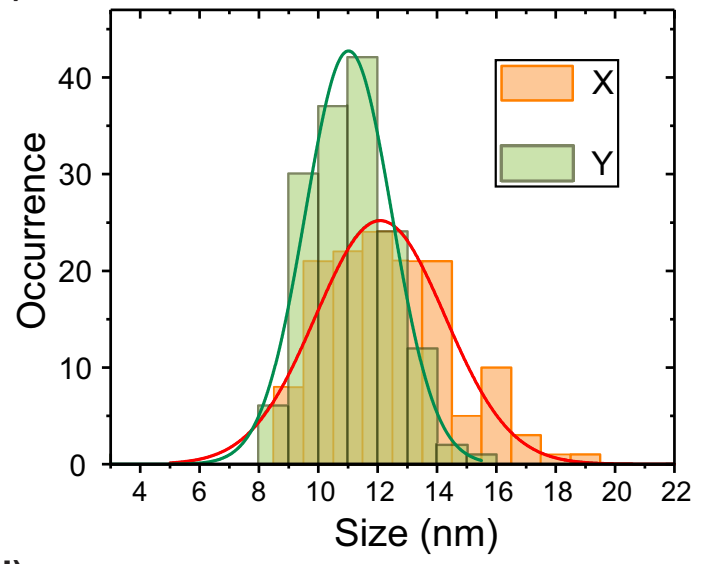

(d)

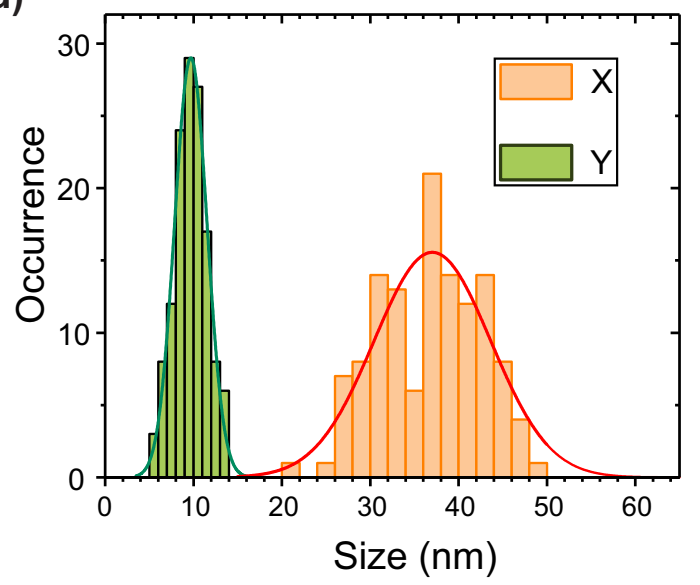

Fig. S. 1: Average NPLs core sizes extracted from TEM images analysis. (a) and (c): TEM images of core structures before crown growing leading to small core (SC) and large core (LC) NPLs respectively. (b) and (d): size distributions are adjusted using normal distributions - using two images for each of the species - leading to average sizes $\left\langle X_{S C}\right\rangle=12.0 \mathrm{~nm}(\mathrm{FWHM}=5.2 \mathrm{~nm})$, $\left\langle Y_{S C}\right\rangle=11.0 \mathrm{~nm}(\mathrm{FWHM}=3.4 \mathrm{~nm})$ and $\left\langle X_{L C}\right\rangle=37.0 \mathrm{~nm}(\mathrm{FWHM}=15.5 \mathrm{~nm}),\left\langle Y_{L C}\right\rangle=$ $9.7 \mathrm{~nm}(\mathrm{FWHM}=4.3 \mathrm{~nm})$. We note the substantial amount of dispersity in the explored samples. 


\section{Large core and small core NPLs size}

(a)

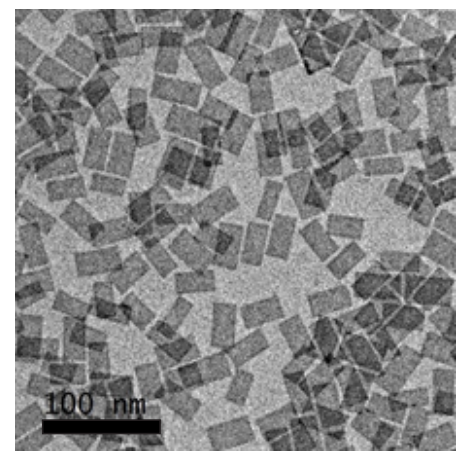

(c)

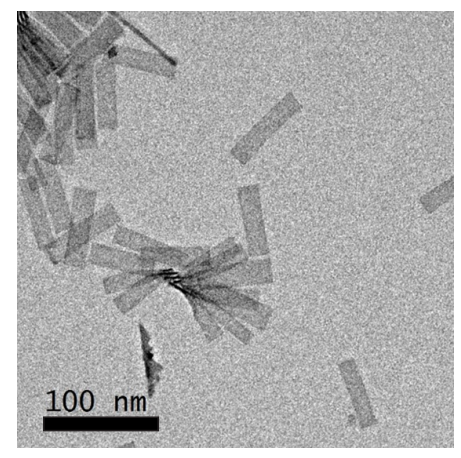

(b)

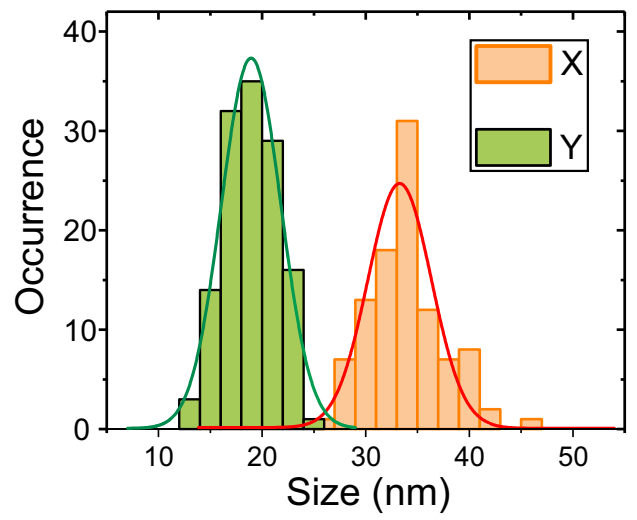

(d)

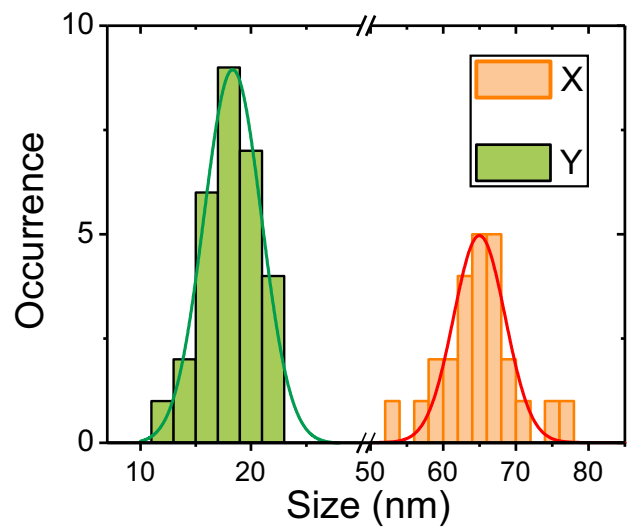

Fig. S. 2: Average core-crown NPLs sizes (LC and SC NPLs) extracted from TEM images analysis. (a) and (c): TEM images of core-crown NPLs hetero-structures following crown-growing (SC and LC NPLs respectively). (b) and (d): size distributions are adjusted using normal distributions leading to average sizes $\left\langle X_{S C}\right\rangle=33.0 \mathrm{~nm}(\mathrm{FWHM}=7.2 \mathrm{~nm}),\left\langle Y_{S C}\right\rangle=19.0 \mathrm{~nm}(\mathrm{FWHM}=$ $6.7 \mathrm{~nm})$ in SC NPLs and $\left\langle X_{L C}\right\rangle=65.0 \mathrm{~nm}(\mathrm{FWHM}=8.3 \mathrm{~nm}),\left\langle Y_{L C}\right\rangle=18.0 \mathrm{~nm}(\mathrm{FWHM}$ $=6.1 \mathrm{~nm}$ ) in LCs NPLs. 


\section{Temperature dependence of the band gap}

A strong shift of the excitonic transitions, $X_{i}$, towards higher energies is observed as the temperature is lowered. The measured variations are typical of II/VI semiconductors. We check here that they can indeed be predicted by the semiempirical Varshny relation that describes the behaviour of the band gap, $E_{g}$, with temperature: ${ }^{1,2}$

$$
E_{g}(T)=E_{g}(0)-\frac{\alpha T^{2}}{\beta+T}
$$

The equation 1 is built in order to account for the variation of two contributions in the energy gap:

- At high temperature the relative position of the conduction band and valence band is due to the dilatation of the lattice. Theoretical calculations show the effect is linear with T.

- The electrons-lattice interaction (major contribution) also changes the gap; this leads to distinct dependences: $\Delta E_{g} \propto T$ above the Debye temperature and $\Delta E_{g} \propto T^{2}$ below the Debye temperature.

$X_{1}$ and $X_{2}$ transitions correspond to the lowest e-hh transition in CdTe and CdSe respectively. Their shifts are plotted in figure S.3. Adjustments of the curves provide $\alpha$ and $\beta$ values in close agreement with the reported values ${ }^{2}$ except for the $X_{2}$ transition (CdSe) in large crown NPLs where the totally unconstrained fit leads to a significantly smaller $\beta$ parameter. We notice that the quality of the fit poorly depends on $\beta$ in a wide range of the parameter value. We find:

- for SC NPLs:

$E_{g, C d T e}(0) \approx 2.337 \pm 0.004 \mathrm{eV} ; \alpha_{C d T e} \approx 0.57 \pm 0.13 \mathrm{meV} / \mathrm{K}$ and $\beta_{C d T e} \approx 166 \pm 107 \mathrm{~K} ;$

$E_{g, C d S e}(0) \approx 2.535 \pm 0.003 \mathrm{eV} ; \alpha_{C d S e} \approx 0.62 \pm 0.22 \mathrm{meV} / \mathrm{K}$ and $\beta_{C d S e} \approx 265 \pm 202 \mathrm{~K} ;$

- for LC NPLs:

$$
\begin{aligned}
& E_{g, C d T e}(0) \approx 2.333 \pm 0.003 \mathrm{eV} ; \alpha_{C d T e} \approx 0.62 \pm 0.17 \mathrm{meV} / \mathrm{K} \text { and } \beta_{C d T e} \approx 199 \pm 133 \mathrm{~K} \\
& E_{g, C d S e}(0) \approx 2.545 \pm 0.004 \mathrm{eV} ; \alpha_{C d S e} \approx 0.50 \pm 0.10 \mathrm{meV} / \mathrm{K} \text { and } \beta_{C d S e} \approx 114 \pm 78 \mathrm{~K}
\end{aligned}
$$



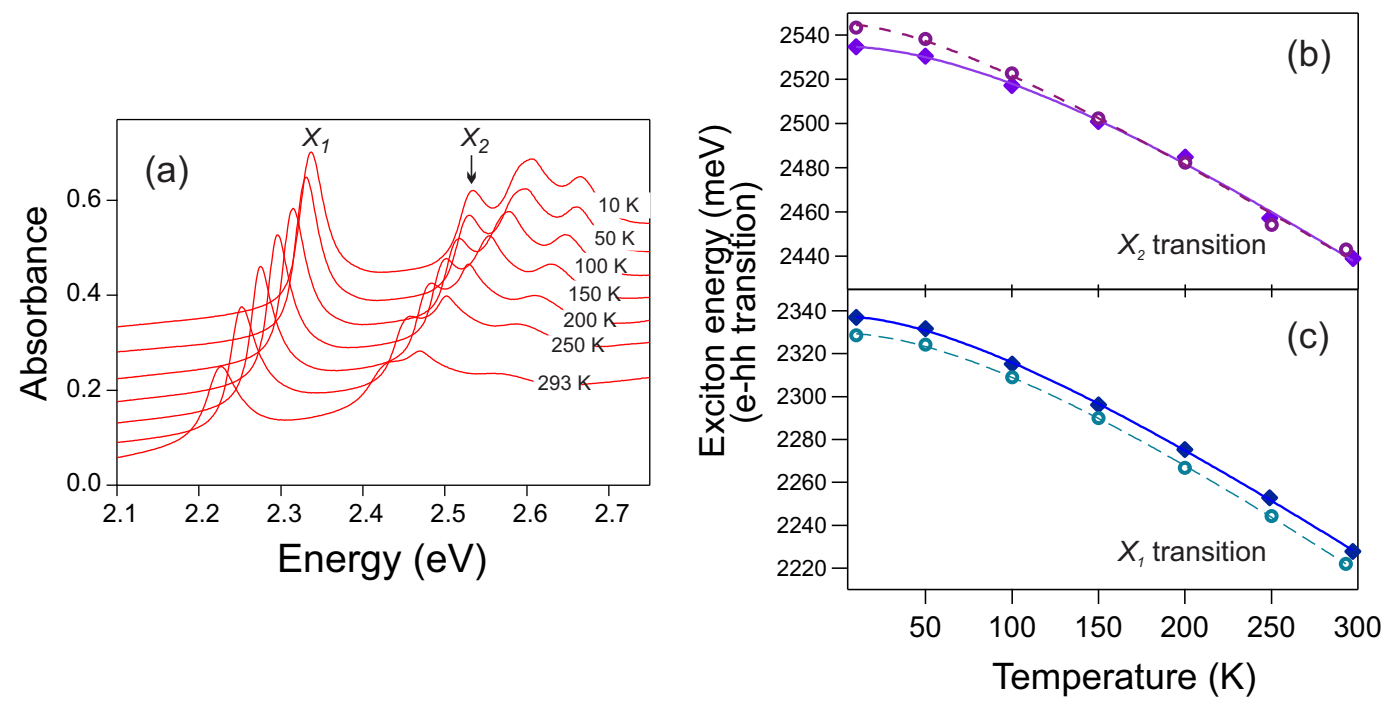

Fig. S. 3: (a) Absorption as a function of temperature (10 K - $293 \mathrm{~K})$ in small core (SC) NPLs showing the characteristic blue shift as $T$ decreases. (b) Energy of the $X_{2}$ resonance (CdSe core) in small core (SC) NPLs (solid diamond) and large core (LC) NPLs (open circle). (c) Energy of the $X_{1}$ resonance (CdTe crown) - legend as in (b).

Reducing the uncertainty on $\alpha$ and $\beta$ parameters would require improved measurements (with reduced uncertainty on $T$, improved spectral resolution and larger set of values). Accurate modelling of the gap evolution in NPLs should in addition account for the confined nature of phonons, a task beyond the scope of the present study. We provide here a rough estimation of the Varshny parameters for 4 monolayers core-crown NPLs having different core sizes and emphasize that the $\approx 100 \mathrm{meV}$ shift observed between room temperature and $10 \mathrm{~K}$ is typical of line displacements reported so far in II/VI semiconductors heterostructures. We finally notice that the smooth evolutions of the $X_{1}$ and $X_{2}$ transition energies are very likely the indication that there is no phase-transition occuring in the system as temperature varies between $4 \mathrm{~K}$ and $300 \mathrm{~K}$.

\section{Effect of dielectric contrast}

In core-crown CdSe/CdTe NPLs the fine structure of indirect excitons was found to be composed of two linearly polarized states associated to dipoles contained in the NPL plane and having orthogonal orientations. ${ }^{3}$ Due to the large difference in the dielectric constant of the 
CdSe and CdTe semiconductor materials and the surrounding medium (vacuum in our low $\mathrm{T}$ experiments) the electric field penetrating the NPL is reduced. This local field effect drastically depends on the nanocrystal geometry and changes the photon coupling to an exciton. ${ }^{4}$ In order to reach a realistic estimation of the field modification in a simplified picture, NPLs are modelled as flattened homogeneous ellipsoids of revolution $\left(\epsilon_{C d S e} \approx \epsilon_{C d T e}=\epsilon_{i n}, \epsilon\right.$ being the relative permittivity) characterized by the semi-axis length $a, b$ and $c$ (in the $X, Y$ and $Z$ directions respectively).

The projection of the electric field inside, $E_{\alpha}^{\text {in }}$, and outside, $E_{\alpha}^{o u t}$, the nanocrystal $(\alpha=X$, $Y, Z)$ are related as:

$$
E_{\alpha}^{\text {in }}=\frac{E_{\alpha}^{\text {out }}}{1+n^{\alpha}(k-1)}
$$

where $k=\epsilon_{\text {in }} / \epsilon_{\text {out }}\left(\epsilon_{\text {out }}=1\right)$ and $n^{\alpha}$ is the depolarisation factor along the direction $\alpha$. Calculations by Landau and Lifshitz provide: ${ }^{5}$

$$
n^{\alpha}=\frac{1}{2} a b c \int_{0}^{\infty} \frac{d s}{\left(s+\beta^{2}\right) \sqrt{\left(s+a^{2}\right)\left(s+b^{2}\right)\left(s+c^{2}\right)}},
$$

with $\beta=\mathrm{a}($ for $\alpha=X)$, b $(\alpha=Y)$ or c $(\alpha=Z)$.

As the exciting field is tuned far away the excitonic resonance the local field effect is considered in the emission process only. The probability to emit light for each level composing the fine structure is reduced by the screening factor, $D_{\alpha}=\left(E_{\alpha}^{\text {in }} / E_{\alpha}^{\text {out }}\right)^{2} .^{4}$

In LCs NPLs, numerical integration of equation 3 with $a=65 \mathrm{~nm}, b=18 \mathrm{~nm}, c=1.3 \mathrm{~nm}$, leads to:

$$
\begin{aligned}
& -n^{X}=0.0093 \text { and } n^{Y}=0.0638\left(\text { with } \epsilon_{\text {in }} / \epsilon_{\text {out }} \approx 10\right) ; \\
& \text { - } \frac{D_{X}}{D_{Y}} \approx 2
\end{aligned}
$$

In SCs NPLs,we find, with $a=33 \mathrm{~nm}, b=19 \mathrm{~nm}, c=1.3 \mathrm{~nm}$ :

$$
\begin{aligned}
& -n^{X}=0.0247 \text { and } n^{Y}=0.0557 ; \\
& \text { - } \frac{D_{X}}{D_{Y}} \approx 1.5 .
\end{aligned}
$$




\section{Low temperature Raman spectroscopy}

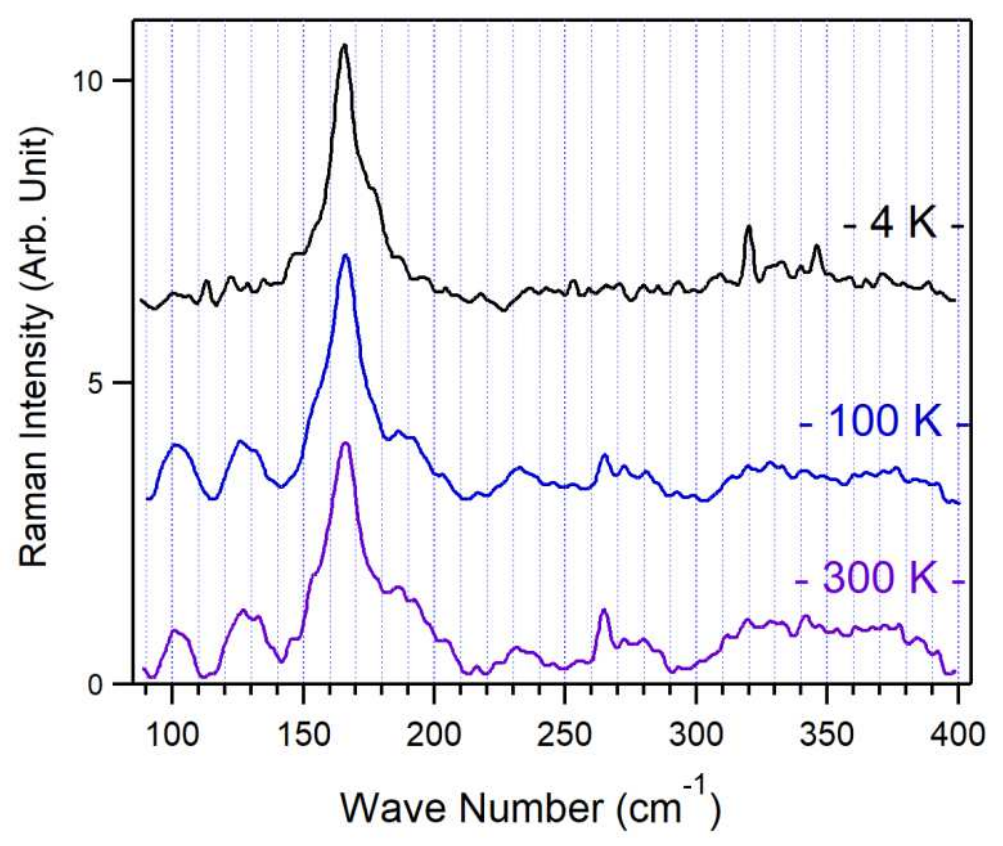

Fig. S. 4: Raman spectrum measured at three temperatures (from top to bottom: $4 \mathrm{~K}, 100 \mathrm{~K}$ and $300 \mathrm{~K}$ ) in CdSe/CdTe core-crown NPLs films (moderate dilution). Raman measurements were conducted by backscattering (T64000, Horiba) a CW diode line (532 nm, $1 \mathrm{~mW}$ ) with a triple stage. Spectra were collected at $>80 \mathrm{~cm}^{-1}$, where the CCD detector (Horiba Synapse Open-Electrode) has a monotonically increasing quantum efficiency of $0.43-0.50$. Acquisitions employed a $100 \times$ optical objective and used minimal laser intensity to avoid sample degradation. The spectrum is dominated by a single peak which position does not move as temperature changes. We report a Raman shift of $166 \pm 2 \mathrm{~cm}^{-1}(\Delta E=20.6 \pm 0.3 \mathrm{meV})$ in agreement with the periodic modulation of single NPLs spectra.

\section{Huang-Rhys factor extraction}

Following the approach of Groeneveld and Donega ${ }^{6}$ the PL lineshape may be modelled as a sum comprising a pure electronic line (zero phonon line or ZPL) and several secondary components peaked at lower energy, (i) each associated to a phonon-assisted emission process (phonon replicae) and, (ii) each displaced in energy by an amount corresponding to multiples of the energy of the phonon involved in the transition. For a transition between the excited excitonic (b) state toward the electronic (a) state, the emission lineshape as a function of 
the energy, $E$, reads (keeping the notation of Groeneveld and coworker ${ }^{6}$ ):

$$
I_{a b}(E)=I_{0} \sum_{n} F_{n}(0) \delta\left(E_{0,0}-n \hbar \omega-E\right)
$$

where $E_{0,0}=E_{b, 0}-E_{a, 0}$ is the energy of the transition between the zero vibrationnal levels of the initial (b) state and final (a) state (ZPL) and $n$ is the number of phonons generated in the transition. $I_{0}$ is the intensity of the full band and $F_{n}(0)$ is the $T=0$ Franck-Condon factor and can be expressed as: ${ }^{7}$

$$
F_{n}(0)=\frac{\exp (-S) S^{n}}{n !}
$$

$S$ being the Huang-Rhys parameter. We note that up to three phonons can be observed in the PL of single core-crown NPLs.

Equation 4 is in principle only valid in the frame of the adiabatic approximation stating that the electron-phonon coupling does not give rise to transition between different electronic configurations which could be observed when there are degenerate states or electronic states with energy spacings comparable to phonon energies. The lattice motion is a coupled system problem however the description in terms of displacements of individual ions can be reduced to a single configurational coordinate model (using normal coordinates) in the harmonic approximation (like in molecular systems). ${ }^{7}$ The electron-phonon interaction Hamiltonian is then taken to be linear in the normal vibrationnal amplitude. The ZPL has the intensity $I_{0} \exp (-S)$ and if $S=0$ all the intensity is contained in the ZPL. As $S$ increases the intensity in the ZPL decreases and this is compensated for by the emergence of vibrational sidebands at energies $n \hbar \omega$ below the ZPL.

Extraction of the Huang-Rhys factor: the single NPLs spectra were adjusted using the progression given by equations 4 and 5 . The $n^{\text {th }}$ vibronic line has a weight $I_{0} F_{n}(0)$ in the band, which is extracted from the adjustment of the experimental curve (see below for procedure). Plotting $F_{n+1}(0) / F_{n}(0)$ as a function of $(n+1)^{-1}$ allows to estimate the value of $S$ (slope of 
the plot). When doing so, we are led to the following conclusions:

- The expected linear dependence is indeed observed in the majority of considered objects (see figure S.5.a-c, e);

- Some deviations are however reported and show up in the basic adjustment of single NPLs spectra (see for instance fit of figure S.5.d);

- $0.8<S<1$ is found in more than $80 \%$ of the studied nanocrystals; we report spectra characterized by $S>1$ but the occurrence is much less frequent;

- Some of the spectra cannot be adjusted reasonably using the progression of equation $4(\approx 10 \%)$ and it was decided they would not contribute to the statistics presented in the study.

Guidelines for the adjustment protocol: the sharpest peaks at higher energy (ZPL and first phonon replica) are first considered and provide most of the guess values injected in the fit:

(1) From their positions in energy we extract the spacing (phonon energy) $\Delta E_{1}=$ $E_{a, 1}-E_{a, 0}=\hbar \omega_{1}, \Delta E_{2}=\hbar \omega_{2}, \ldots \Delta E_{p}$. When peaks do not show up for $p>2$, the transition energies, $E_{n}$, were generated as follows: $E_{n}=E_{b, 0}-E_{a, n} \approx E_{0,0}-n \hbar \omega_{2}$;

(2) The ratio of the amplitudes provides a first estimation of the Huang-Rhys factor, $S=F_{1} / F_{0}$. As a first step the weighting coefficients, $F_{n}$, are imposed by equation 5 . They can be further optimized through the fit procedure, as a second step.

(3) As a first approximation correct fits are obtained by imposing identical linewidths for all phonon sidebands. It is however clear that some of the ZPLs are found to have slightly narrower linewidths. 

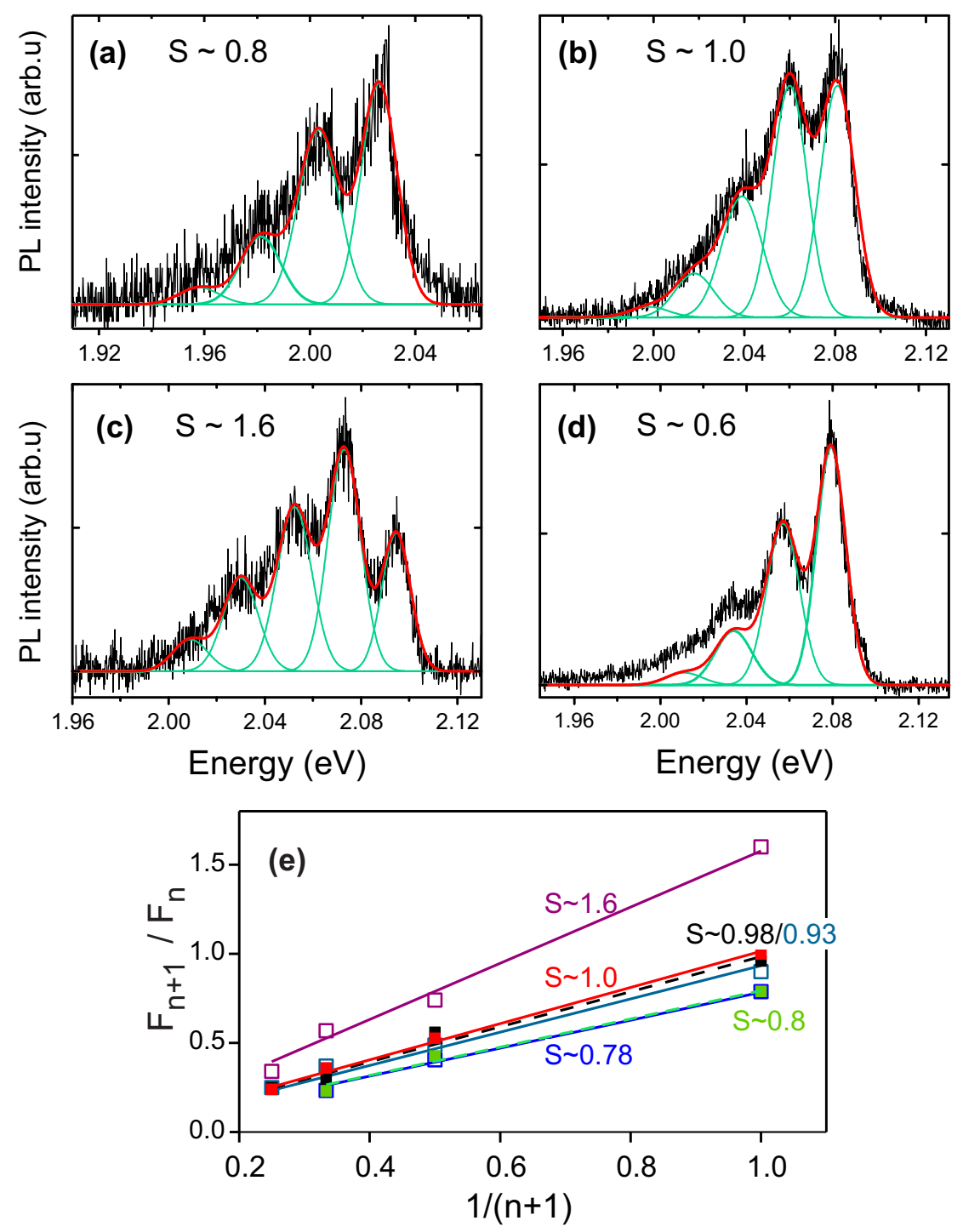

Fig. S. 5: Typical PL spectra from single CdSe/CdTe core-crown NPLs with different HuangRhys factors: (a) $S \approx 0.8$, (b) $S \approx 1.0$, (c) $S \approx 1.6$ and (d) $S \approx 0.6$; in the latter case the adjustment, based on the ZPL - first replica amplitude ratio, is clearly less good, showing a slight underestimation of the fit on the low energy side. Most of the studied NPLs ( $\geq 80 \%$ in number) had a Huang-Rhys factor around $0.8-1$. The green curves represent the components (ZPL and phonon replicae) from which the relative amplitude $F_{n}$ are extracted (see text). (e) Plot of the $F_{n+1} / F_{n}$ ratio as a function of $(n+1)^{-1}$ in six NPLs. The linear dependence is expected for a phonon progression in the displaced oscillator model; the slope provides the Huang-Rhys factor, $S$.

\section{References}

(1) Varshny, Y. P. Temperature Dependence of the Energy Gap in Semiconductors. Physica 1967, 34, 149-154. 
(2) Tamargo, M., Ed. II-VI Semiconductor Materials and their Applications; Optoelectronic Properties of Semiconductors and Superlattices; CRC Press, 2002; Vol. 12.

(3) Pandya, R. et al. Fine Structure and Spin Dynamics of Linearly Polarized Indirect Excitons in Two-Dimensional CdSe/CdTe Colloidal Heterostructures. ACS Nano 2019, 13, $10140-10153$.

(4) Rodina, A.; Efros, A. L. Effect of Dielectric Confinement on Optical Properties of Colloidal Nanostructures. J. Exp. Theor. Phys. 2016, 122, 554-566.

(5) Landau, L.; Lifshitz, E. M. Course of theoretical Physics, Vol. 8: Electrodynamics of Continuous Media; Pergamon: Oxford, 1960.

(6) Groeneveld, E.; de Mello Donegá, C. Enhanced Exciton-Phonon Coupling in Colloidal Type-II CdTe-CdSe Heteronanocrystals. J. Phys. Chem. C 2012, 116, 16240-16250.

(7) Henderson, B.; Imbusch, G. Optical Spectroscopy of Inorganic Solids; Oxford University Press, 2006. 